\title{
ESTRUCTURA POBLACIONAL Y CARACTERÍSTICAS DEL HÁBITAT DE dos especies de Cyatheaceae del estado de Hidalgo, México
}

\author{
María Guadalupe Pérez-Paredes ${ }^{1}$, Arturo Sánchez-González ${ }^{1,3}$ y José Daniel Tejero-Díez² \\ ${ }^{1}$ Centro de Investigaciones Biológicas, Universidad Autónoma del Estado de Hidalgo, \\ Mineral de la Reforma, Hidalgo, México \\ ${ }^{2}$ Facultad de Estudios Superiores Iztacala, Universidad Nacional Autónoma de México. \\ Tlalnepantla, Estado de México, México \\ ${ }^{3}$ Autor para la correspondencia: arturosg@uaeh.edu.mx
}

\begin{abstract}
Resumen: Los helechos arborescentes (Cyatheaceae) constituyen un componente conspicuo del bosque húmedo de montaña de las regiones tropicales y subtropicales del mundo. En México su distribución se circunscribe casi por completo al bosque húmedo de montaña, y pese a que algunas de las especies se incluyen dentro de la Norma Oficial Mexicana y en las listas rojas internacionales, no se cuenta con información sobre el estado actual de sus poblaciones. En el presente estudio se generó información básica acerca de la estructura, densidad poblacional y las características del hábitat de Alsophila firma y Cyathea fulva para establecer en qué grado las condiciones actuales de su hábitat ponen en riesgo su supervivencia. Estas dos especies son de amplia distribución dentro del bosque húmedo de montaña, consideradas como "sujetas a protección especial” en la legislación mexicana. Se encontró que las poblaciones de ambas especies son normales o dinámicas, con alto potencial de regeneración, formando parte del sub-dosel en sitios con cobertura arbórea alta, por lo que son tolerantes a la sombra. Las variables que mejor explicaron las diferencias en la densidad poblacional fueron la altitud, la pendiente, la presencia de caminos y la cobertura del suelo para A. firma; mientras que en $C$. fulva fue la cobertura del dosel. El establecimiento y supervivencia de las poblaciones de ambas especies depende en gran medida de la conservación de su hábitat, debido a que requieren de la existencia de un alto porcentaje de cobertura vegetal.
\end{abstract}

Palabras clave: Alsophila firma, bosque húmedo, Cyathea fulva, estructura de tamaños, riesgo de extinción.

\begin{abstract}
The tree ferns (Cyatheaceae) are a conspicuous component of the humid montane forest in the tropical and subtropical regions of the world. In Mexico, the tree ferns distribution is limited almost entirely to the humid montane forest (cloud forest). Although some species are included in the Norma Oficial Mexicana (governmental regulations normalized) and international red lists, there is no information on the current status of their populations. Two species widely distributed within the montane rainforest, and classified as "subject to special protection" under Mexican law, are Alsophila firma and Cyathea fulva. In the present study, basic data was collected about the structure, population density and habitat characteristics of these tree ferns, in order to determine to what extent the current state of their habitat threatens their survival. We found that populations of both species are normal or dynamic, with high regeneration potential, forming part of the sub-canopy at sites with high tree cover, making them tolerant to shade. The variables that best explained differences in population density for A. firma were elevation, slope, presence of roads, and ground cover, while for C. fulva it was canopy cover. The establishment and survival of populations of both species depends heavily from conservation of their habitat (humid montane forest) because they require a high percentage of vegetation cover.
\end{abstract}

Key words: Alsophila firma, Cyathea fulva, extinction risk, humid forest, size structure.

$\mathbf{L}$ os helechos arborescentes (Cyatheaceae), con su característico tallo y grandes hojas, constituyen un componente conspicuo del bosque húmedo de montaña en las regiones subtropicales del planeta (Large y Braggins, 2004; Bystriakova et al., 2011). En la República Mexicana, siguen un patrón de distribución montano, principalmente en la vertiente de sotavento de la Sierra Madre del Sur y
Sierra Madre Oriental, y en los extremos oceánicos de la Faja Volcánica Transmexicana. Se encuentran en ambientes de atmósfera saturada de humedad, como es el bosque húmedo de montaña (BHM), donde tienen una distribución más o menos homogénea y llegan a constituirse como parte importante del estrato arbóreo inferior (Ramírez-Barahona et al., 2011). 
El BHM en México es un ecosistema con una distribución fragmentaria y limitada a una estrecha faja en la zona montañosa, ocupando preferentemente un intervalo altitudinal que va desde los 1,000 a los 2,500 m (Villaseñor, 2010). Se desarrolla en sitios con características geográficas y ecológicas heterogéneas, con pendientes pronunciadas y en laderas expuestas al viento, en donde es común la alta disponibilidad de agua y humedad atmosférica durante todo el año (Rzedowski, 2006; Villaseñor, 2010). Actualmente se encuentra seriamente amenazado por la actividad antrópica en la explotación forestal y el cambio de uso del suelo, y posee una de las tasas de deforestación más altas entre los bosques templados de México (Villaseñor, 2010; Cruz-Cárdenas et al., 2012; Ponce-Reyes et al., 2012).

La mayoría de las especies de Cyatheaceae se encuentran amenazadas y se incluyen en alguna categoría de riesgo dentro de las listas rojas internacionales (IUCN, 2011) y la Norma Oficial Mexicana NOM-059 (SEMARNAT, 2010). En México, Alsophila firma y Cyathea fulva son dos especies con amplia distribución dentro del BHM y están consideradas en la categoría de "sujetas a protección especial" en la legislación mexicana, por lo que es necesario que se generen datos biológicos a nivel poblacional para tener la certeza de que el uso, extracción y comercialización de los individuos de las dos especies están adecuadamente fundamentados, y que no se ponga en riesgo su supervivencia (Mehltreter y García-Franco, 2008; CITES, 2010).

Al conocer la estructura poblacional (v. gr. la distribución de frecuencias en las distintas categorías demográficas de los individuos y las clases de edad que están mejor representadas), y la fenología (Vandermeer y Goldberg, 2003), se pretende averiguar la dinámica de las poblaciones, aspecto que es útil y necesario cuando se trata de especies que están incluidas en alguna categoría de riesgo, para establecer estrategias de manejo y conservación a corto, mediano y/o largo plazo (Schmitt y Windisch, 2006; Aguraiuja et al., 2008; Mehltreter, 2010).

En el continente Americano se han realizado un número considerable de investigaciones acerca de la estructura poblacional y la fenología de varias especies de helechos arborescentes, que indican que existe gran variación entre especies y entre hábitats, con respecto a características tales como: producción y germinación de esporas, fenología foliar, tasa de crecimiento, edad de los individuos, preferencias de hábitat, tolerancia al disturbio, entre otras (Bernabe et al., 1999; Arens y Sánchez, 2000; Schmitt y Windisch, 2006; Mehltreter y García-Franco, 2008; Sharpe y Mehltreter, 2010; Bystriakova et al., 2011; Schmitt y Windisch, 2012). En el caso de México, se han realizado pocos esfuerzos en el tema y sólo es posible resaltar el estudio de la fenología foliar y el crecimiento de los individuos de una población de Alsophila firma, realizado por Mehltreter y García-Franco (2008).

Ante la carencia de información, a nivel poblacional, de las especies mexicanas de Cyatheaceae y, debido a la nece- sidad de conocer más sobre varios aspectos fundamentales de la biología de las especies de plantas que se encuentran en alguna categoría de protección, en el presente estudio se plantearon los siguientes objetivos: (1) generar información básica acerca de la estructura poblacional de dos especies de helechos arborescentes (Alsophila firma y Cyathea ful$v a)$ que se desarrollan en el BHM del estado de Hidalgo, tomando como referencia dos municipios representativos de su área de distribución; y (2) caracterizar el hábitat de las dos especies, para definir cuáles son las variables ambientales más estrechamente relacionadas con la densidad y distribución de sus poblaciones.

\section{Material y métodos}

Se localizaron poblaciones de Alsophila firma y Cyathea fulva en la zona montana del estado de Hidalgo, en varias localidades con BHM de los municipios de San Bartolo Tutotepec y Tlanchinol (Figura 1), en donde previamente, mediante exploración en campo, se habían identificado individuos de ambas especies (Álvarez-Zúñiga et al., 2012; Pérez-Paredes et al., 2012).

Tlanchinol. Se ubica al norte del estado de Hidalgo, entre las coordenadas $20^{\circ} 54^{\prime}$ y $21^{\circ} 12^{\prime}$ de latitud norte y $98^{\circ} 30^{\prime}$ y $98^{\circ} 45^{\prime}$ de longitud oeste. El intervalo de altitud va de 200 a $1,900 \mathrm{~m}$, y ocupa $1.88 \%$ de la superficie estatal. Presenta un clima semicálido húmedo con lluvias todo el año y templado húmedo con lluvias todo el año. La precipitación promedio anual es de $2,156.2 \mathrm{~mm}$ y la temperatura promedio de $17.2^{\circ} \mathrm{C}$. La mayor parte del municipio está cubierto por BHM (38\%) y en menor proporción por bosque tropical (21\%), el resto está destinado para uso de suelo agrícola y habitacional (INEGI, 2009; Pavón y Meza, 2009; ÁlvarezZúniga et al., 2012).

San Bartolo Tutotepec. Se localiza entre las coordenadas $20^{\circ} 19^{\prime}$ y $20^{\circ} 37^{\prime}$ de latitud norte y $98^{\circ} 02^{\prime}$ y $98^{\circ} 21^{\prime} \mathrm{de}$ longitud oeste; el intervalo altitudinal va de 300 a 2,600 m, y ocupa el $1.72 \%$ de la superficie del estado. El clima dominante es semicálido húmedo con lluvias todo el año, y en menor proporción templado húmedo con lluvias todo el año, templado húmedo con abundantes lluvias en verano y semifrío húmedo con abundantes lluvias en verano. La precipitación promedio anual es de aproximadamente $1,655.9 \mathrm{~mm}$ y la temperatura promedio de $17.2{ }^{\circ} \mathrm{C}$. Los principales tipos de vegetación son BHM (33\%), bosque tropical $(13 \%)$ y pastizal (5\%), mientras que el $49 \%$ restante es ocupado por cultivos y zonas urbanas (Rzedowski, 2006; Pavón y Meza, 2009; Rodríguez-Ramírez et al., 2013).

Estructura poblacional. Para el análisis de la estructura poblacional de Alsophila firma y Cyathea fulva se eligieron cuatro poblaciones de cada especie: dos ubicadas en el municipio de San Bartolo Tutotepec y dos en el de Tlanchinol, estado de Hidalgo. Los municipios elegidos representan dos 


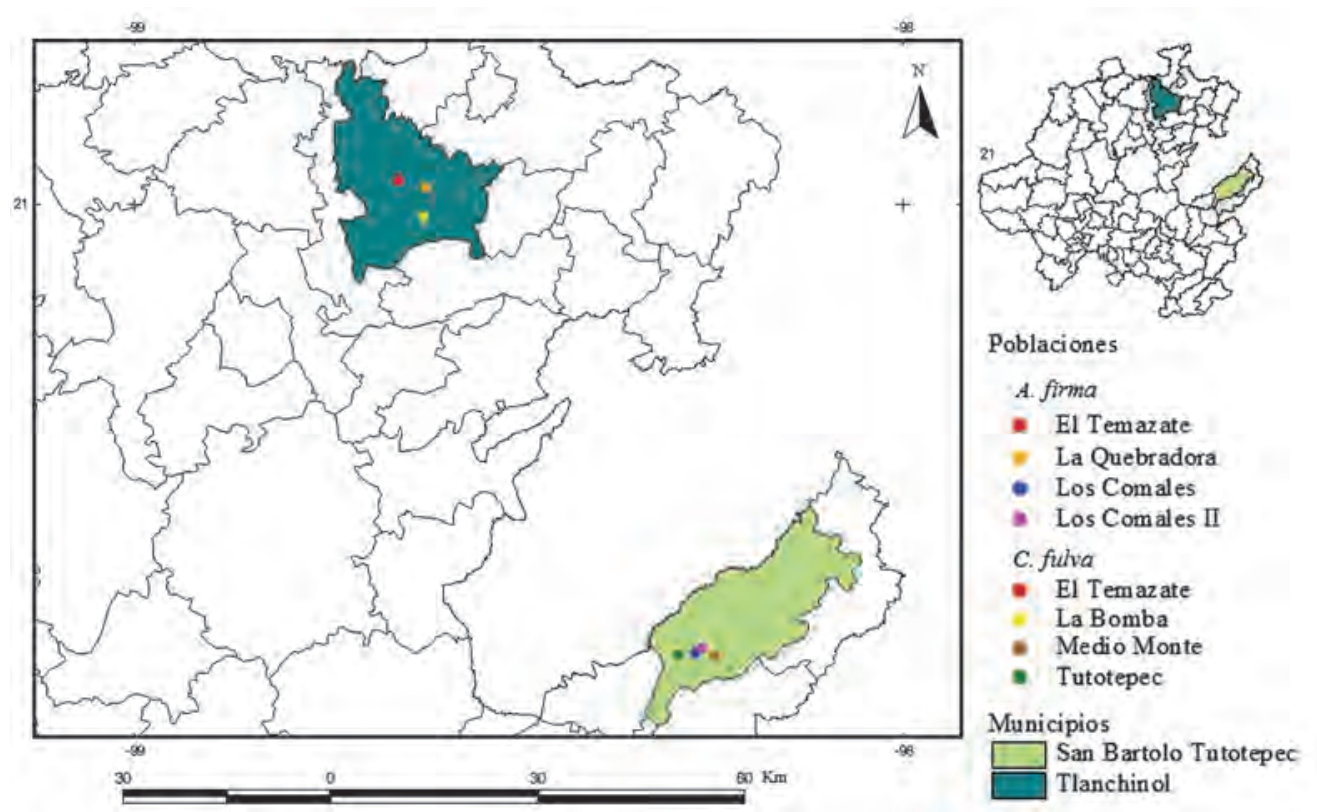

Figura 1. Área de estudio

puntos extremos y representativos de la distribución del bosque húmedo de montaña en la entidad.

En el presente estudio se definió a una población local, como un grupo de individuos que viven en el mismo entorno dentro de un hábitat y por consiguiente, experimentan condiciones ambientales similares (Hanski y Simberloff, 1997; Aguraiuja et al., 2008). De acuerdo con esta definición, el criterio de selección de las poblaciones se basó en la presencia de al menos diez individuos, en sitios en donde el tamaño de fragmento de bosque permitiera establecer las parcelas de muestreo bajo el dosel, evitando así el efecto de borde. Los sitios seleccionados presentaron en general "buen estado de conservación", dado que durante los recorridos de campo no se observaron poblaciones de más de cinco individuos en sitios abiertos (sin cobertura arbórea) o perturbados, únicamente organismos aislados en ambas especies.

En cada población seleccionada se colocaron tres parcelas de muestreo de $400 \mathrm{~m}^{2}$ cada una, separadas entre sí por al menos una distancia de $20 \mathrm{~m}$; por lo que el número total de parcelas para el análisis de las cuatro poblaciones de cada especie fue de 12 (seis parcelas por municipio). El tamaño de la unidades de muestreo es el sugerido para la caracterización poblacional en algunas especies de helechos arborescentes (Schmitt et al., 2009; Alfonso-Moreno et al., 2011).

Dentro de cada parcela de muestreo se contabilizaron las siguientes variables poblacionales: (1) densidad de individuos, cada tallo con una corona de frondas se consideró como un individuo; (2) altura del tallo de cada individuo; (3) diámetro normal del tallo a la altura del pecho (DAP a $130 \mathrm{~cm}$, o en la parte apical del tallo en individuos de menor altura); (4) número de individuos maduros y fértiles; y (5) número de hojas (Kingston et al., 2004; Jones et al., 2007). Los valores obtenidos para cada una de las variables (excepto los de densidad de individuos) en las diferentes poblaciones fueron contrastados con análisis de varianza de una vía con la prueba de Tukey de comparación múltiple de medias, utilizando el programa Statistica versión 9 (StatSoft Inc., 2010).

Como aún es controversial la relación entre la fertilidad y la edad en varias especies de helechos arborescentes, la altura del tallo de los individuos continúa siendo un criterio ampliamente utilizado para definir la estructura por edades de las poblaciones (Nagano y Susuki, 2007; Mehltreter y García-Franco, 2008; Schmitt y Windisch, 2012). En el presente estudio se utilizaron los valores de densidad y altura del tallo de los individuos para definir la estructura poblacional de cada especie y poder así clasificarlas, con base en una simplificación del criterio propuesto por Aguraiuja et al. (2008) en dinámicas, normales o regresivas. Se estableció que una población dinámica contenía un elevado número de individuos de talla pequeña (más de 65\% de los organismos de menos de $3 \mathrm{~m}$ de altura), y un número menor de individuos de tamaño intermedio (de entre 3 y $7 \mathrm{~m}$ de altura) y alto (de más de $7 \mathrm{~m}$ ); una población normal o estándar se caracterizó por la presencia de individuos de todas las clases de tamaño, pero con predominio de los de altura intermedia (35-65\% de los organismos); y una población regresiva, por contener un número elevado de individuos altos (más de $65 \%$ ), y un escaso número de estatura baja y/o intermedia.

Estudio de la fenología. Con el fin de conocer la periodicidad en la producción y el tiempo de vida de las hojas, se seleccionaron y marcaron al azar 20 individuos de Cyathea 
fulva en el municipio de San Bartolo Tutotepec, y 20 individuos de Alsophila firma en el municipio de Tlanchinol. A cada uno de los individuos marcados se les midieron en cuatro ocasiones entre los años 2011 y 2012, las siguientes características: altura, DAP, número de hojas (nuevas, maduras y senescentes) y número de pecíolos y cicatrices foliares. Debido a que en la mayoría de las especies de $\mathrm{Al}$ sophila y Cyathea que se han estudiado, se ha estimado que la vida media de las hojas es de más de 10 meses (Schmitt y Windisch, 2006; Mehltreter y García-Franco, 2008; Sharpe y Mehltreter, 2010; Schmitt y Windisch, 2012), en el presente estudio se consideró adecuado un intervalo de tiempo entre mediciones de entre cinco y seis meses.

Un análisis de correlación lineal simple permitió estimar el grado de relación entre las variables: altura del tronco, DAP y número de cicatrices foliares; utilizando el programa de cálculo Statistica versión 9 (StatSoft Inc., 2010). Se utilizaron dos criterios para definir la edad aproximada de los individuos: uno convencional, que consiste en primer lugar en estimar la tasa de crecimiento promedio anual a partir de la diferencia entre el promedio de altura inicial y final de los tallos de los individuos, en un intervalo de 12 meses; y posteriormente, en dividir la altura de los tallos, entre la tasa de crecimiento promedio anual (Sharpe y Mehltreter, 2010). En el segundo criterio, se multiplica el número de cicatrices foliares de cada individuo por la "longevidad de las hojas". De acuerdo con algunos autores, el estudio de la fenología foliar en helechos arborescentes requiere de varios años de duración, porque la producción de las hojas es asincrónica (Schmitt y Windisch, 2006; Mehltreter y García Franco, 2008). Dado que la presente investigación abarcó un periodo de tiempo relativamente corto: 16 meses en $\mathrm{Al}$ sophila firma y 19 meses en Cyathea fulva, se consideró como un valor aproximado de la longevidad al número total de meses que transcurrieron entre dos periodos de máxima producción de hojas nuevas o brotes.

Caracterización del hábitat. Consistió en la descripción de la estructura de la vegetación, con base en las especies dominantes (sensu Rzedowski, 2006), y en la estimación de las siguientes variables ambientales: altitud $(\mathrm{m})$ utilizando un GPSMap 60CS Garmin; cobertura total del dosel (\%), por medio de un densitómetro de espejo cóncavo; orientación (puntos cardinales) utilizando una brújula; y pendiente (grados), calculada con un clinómetro marca Suunto. Adicionalmente, con la finalidad de establecer si existe relación entre la densidad poblacional y las condiciones del hábitat, en cada parcela se estimó el grado de perturbación por medio de las siguientes variables: número de caminos y/o brechas, distancia a sitios de actividad humana (poblados) y vías de comunicación (carreteras, caminos de terracería); cobertura de rocas (\%), hierbas y arbustos recurrentes; indicios de incendios, extracción de plantas, tala de árboles y deslaves evidentes (Cuadro 1).
La relación entre la densidad de las poblaciones de cada especie y los factores ambientales antes mencionados se analizó mediante técnicas de análisis no lineal (árboles de regresión), con el programa de cálculo Statistica versión 9 (StatSoft Inc., 2010). Los árboles de regresión no hacen suposiciones a priori sobre algún tipo de comportamiento entre las variables estudiadas, por lo que son útiles para exponer relaciones no lineales, lo que facilita la interpretación de los resultados (Sánchez-González y López-Mata, 2005; Kallimanis et al., 2007). El algoritmo de los árboles de regresión fue diseñado para realizar particiones recursivas de los datos, generando subgrupos cada vez más homogéneos; en cada división se elige de entre las variables independientes (categóricas y/o continuas), a la que produce como valor umbral la mayor reducción en la suma de cuadrados del error para la variable dependiente. El modelo resultante está estructurado desde un nodo inicial (raíz), que se continúa a través de una serie de divisiones binarias de las variables independientes o explicativas (tallos), hasta los nodos terminales (hojas). La estimación para todas las observaciones que siguen la misma vía desde la raíz hasta una determinada hoja es el valor promedio de la variable dependiente para ese subconjunto de observaciones (Sánchez-González y López-Mata, 2005).

\section{Resultados}

Estructura de tamaños de las poblaciones. La mayoría de los individuos de Alsophila firma en la localidad de El Temazate $(n=138)$ pertenecen a clases de tamaño pequeñas (plantas jóvenes con tallos de menos de $3 \mathrm{~m}$ de alto), pocos son de tamaño intermedio (miden entre 3 y $7 \mathrm{~m}$ de altura) y no se encontraron individuos altos (tallos de más de $7 \mathrm{~m}$; Figura 2A), por lo que la población puede considerarse como dinámica. En La Quebradora $(n=203)$, la distribución de tamaños es similar, excepto por la presencia de dos individuos de más de $7.5 \mathrm{~m}$ de altura (Figura 2B). En la población de Los Comales $(n=36)$, la distribución de tamaños es más homogénea (población normal), la mayoría de los individuos son de estatura baja e intermedia y sólo tres miden $8 \mathrm{~m}$ o más (Figura 2C). En Los Comales II $(\mathrm{n}=10)$ nueve de los individuos fueron pequeños (población dinámica), uno fue de tamaño intermedio y no se observaron individuos altos (Figura 2D).

En el caso de Cyathea fulva, la mayoría de los individuos de la población de El Temazate $(n=68)$ presentan tallos de tamaño intermedio (población normal), y el número disminuye en ambos extremos de la distribución (Figura 2E): pocos individuos son de tamaño pequeño y sólo dos presentan tallos de $8 \mathrm{~m}$ o más. En la población de La Bomba ( $\mathrm{n}=$ 34), la distribución de tamaños es un poco más homogénea (población normal); aunque la mayoría es de talla intermedia, también están representados organismos de estaturas bajas y altas (Figura 2F). En la población de Medio Monte 
Cuadro 1. Características del hábitat de las especies de Cyatheaceae en el estado de Hidalgo. Parcelas 1-6 pertenecen al municipio de Tlanchinol, y del 7-12 al municipio de San Bartolo Tutotepec. P = parcelas; NI = Número de individuos; SV = Suelo con vegetación (\%); VA = Caminos, estimados como la sumatoria de las vías de acceso al sitio, a las cuales se les asignó un peso diferencial (con pavimento, terracería, vereda); CR $=$ Cobertura de rocas (\%); $\mathrm{AH}=$ Distancia a sitios de actividad humana (min.); $\mathrm{ED}=$ Evidencia de deslave (presencia-ausencia); $\mathrm{CD}=\mathrm{Cobertura}$ de dosel $(\%) ; A L T=$ Altitud $(\mathrm{m}) ; \mathrm{PE}=$ Pendiente $\left({ }^{\circ}\right) ; \mathrm{O}=$ Orientación

\begin{tabular}{|c|c|c|c|c|c|c|c|c|c|c|}
\hline $\mathrm{P}$ & $\mathrm{NI}$ & SV & VA & $\mathrm{CR}$ & $\mathrm{AH}$ & ED & $\mathrm{CD}$ & ALT & $\mathrm{PE}$ & $\mathrm{O}$ \\
\hline \multicolumn{11}{|c|}{ Alsophila firma } \\
\hline 1 & 27 & 60 & 65.6 & 2 & 40 & 1 & 98.4 & 1,459 & 55 & $\mathrm{~N}$ \\
\hline 2 & 86 & 70 & 65.6 & 0 & 40 & 1 & 99.7 & 1,459 & 45 & $\mathrm{~N}$ \\
\hline 3 & 25 & 65 & 65.6 & 6 & 40 & 1 & 98.9 & 1,459 & 45 & $\mathrm{~N}$ \\
\hline 4 & 45 & 30 & 59.4 & 4 & 60 & 1 & 97.1 & 1,321 & 50 & $\mathrm{~N}$ \\
\hline 5 & 80 & 30 & 59.4 & 15 & 60 & 1 & 98.8 & 1,321 & 50 & $\mathrm{~N}$ \\
\hline 6 & 80 & 40 & 59.4 & 14 & 60 & 1 & 97.9 & 1,321 & 50 & $\mathrm{~N}$ \\
\hline 7 & 11 & 65 & 40.6 & 10 & 40 & 0 & 98.4 & 1,877 & 60 & $\mathrm{SO}$ \\
\hline 8 & 9 & 30 & 40.6 & 0 & 40 & 0 & 97.1 & 1,877 & 60 & SE \\
\hline 9 & 16 & 25 & 40.6 & 10 & 40 & 0 & 97.1 & 1,877 & 60 & SE \\
\hline 10 & 4 & 40 & 37.5 & 5 & 30 & 0 & 95.6 & 1,939 & 30 & $\mathrm{NO}$ \\
\hline 11 & 3 & 50 & 37.5 & 0 & 30 & 0 & 95.8 & 1,939 & 30 & $\mathrm{NO}$ \\
\hline 12 & 3 & 45 & 37.5 & 0 & 30 & 0 & 93.6 & 1,939 & 30 & $\mathrm{NO}$ \\
\hline \multicolumn{11}{|c|}{ Cyathea fulva } \\
\hline 1 & 20 & 60 & 65.6 & 2 & 40 & 1 & 98.4 & 1,459 & 55 & $\mathrm{~N}$ \\
\hline 2 & 8 & 70 & 65.6 & 0 & 40 & 1 & 99.7 & 1,459 & 45 & $\mathrm{~N}$ \\
\hline 3 & 40 & 65 & 65.6 & 6 & 40 & 1 & 98.9 & 1,459 & 45 & $\mathrm{~N}$ \\
\hline 4 & 11 & 55 & 59.3 & 0 & 10 & 0 & 99.2 & 1,361 & 30 & $\mathrm{NE}$ \\
\hline 5 & 12 & 65 & 59.3 & 0 & 10 & 0 & 98.7 & 1,361 & 30 & $\mathrm{NE}$ \\
\hline 6 & 11 & 55 & 59.3 & 0 & 10 & 0 & 99.2 & 1,361 & 30 & $\mathrm{NE}$ \\
\hline 7 & 22 & 65 & 31.2 & 0 & 5 & 0 & 95.6 & 1,751 & 30 & $\mathrm{NO}$ \\
\hline 8 & 16 & 40 & 31.2 & 5 & 6 & 0 & 92.2 & 1,751 & 35 & $\mathrm{NO}$ \\
\hline 9 & 29 & 55 & 31.2 & 0 & 6 & 0 & 89.9 & 1,751 & 35 & $\mathrm{NO}$ \\
\hline 10 & 19 & 50 & 28.1 & 20 & 25 & 1 & 98.4 & 1,870 & 45 & $\mathrm{NO}$ \\
\hline 11 & 16 & 40 & 28.1 & 30 & 20 & 0 & 91.4 & 1,870 & 40 & $\mathrm{NO}$ \\
\hline 12 & 8 & 45 & 28.1 & 20 & 20 & 0 & 93.6 & 1,870 & 50 & SE \\
\hline
\end{tabular}

$(\mathrm{n}=67)$, la distribución de tamaños es sesgada: la mayoría de las plantas son de tamaño pequeño (población dinámica), pocas son de estatura intermedia y no se encontraron individuos altos, de más de 7 m (Figura 2G); el mismo patrón de distribución se detectó en la población de Tutotepec $(n=43)$ (Figura $2 \mathrm{H}$ ). La producción de esporas en las poblaciones de ambas especies no mostró alguna relación evidente con respecto a la altura del tallo de los individuos.

De acuerdo con las variables evaluadas, el número de individuos fue considerablemente mayor en las dos poblaciones ubicadas en el municipio de Tlanchinol; la población de Alsophila firma de Los Comales presentó los organismos con tallos más altos $(368 \pm 45 \mathrm{~cm})$, y la de los Comales II los de tallos más pequeños; el DAP no difirió de forma significativa entre poblaciones; el mayor número promedio de hojas o frondas se encontró en las poblaciones ubicadas en Los Comales y Los Comales II (Figura 3A-D).

En el caso de las poblaciones de Cyathea fulva, las que presentaron un número elevado de individuos fueron El Te- mazate y Medio Monte (Figura 3 E-H); la proporción de plantas estériles y fértiles fue diferente en cada población, y en la localidad de Tutotepec no se detectaron individuos con frondas fértiles; en las localidades de El Temazate y La Bomba se encontraron los organismos más altos $(420 \pm 23 \mathrm{~cm}$ y $512 \pm 38 \mathrm{~cm}$, respectivamente) y con mayor número de hojas (18 \pm 0.85 y $20.76 \pm 1.03$, respectivamente); y en El Temazate los de mayor DAP $(20.76 \pm 0.86 \mathrm{~cm})$.

La correlación entre la altura y el diámetro normal (DAP a $130 \mathrm{~cm}$ ) de los individuos fue positiva y estadísticamente significativa $(P<0.01)$, para todas las poblaciones de Cyathea fulva y en tres de las cuatro poblaciones de Alsophila firma. La excepción fue la población de Los Comales II, lo cual puede deberse a un sesgo provocado por el escaso número de plantas de A. firma $(\mathrm{n}=10)$ que se desarrollan en este sitio (Figura 3). Es importante resaltar que, durante el presente estudio, sólo se detectó la muerte de un individuo de $C$. fulva, en la localidad El Temazate. 
A
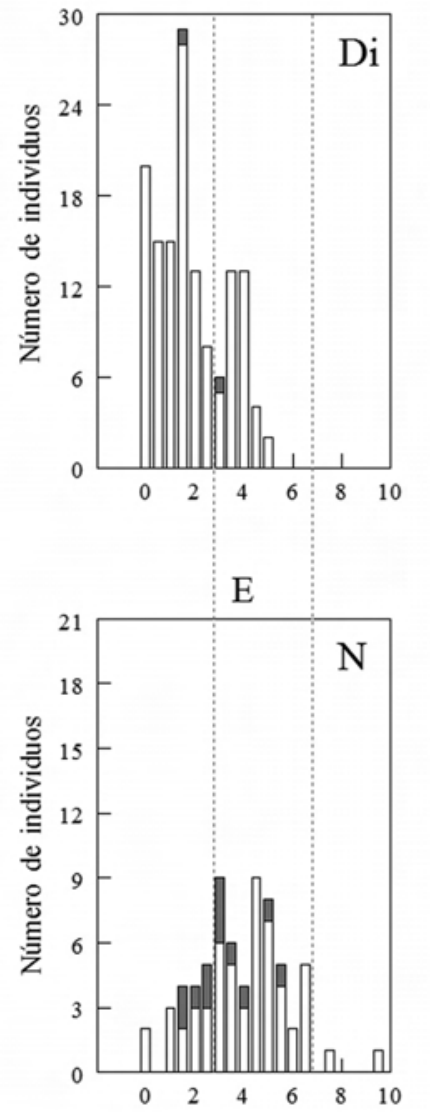

Alsophila firma
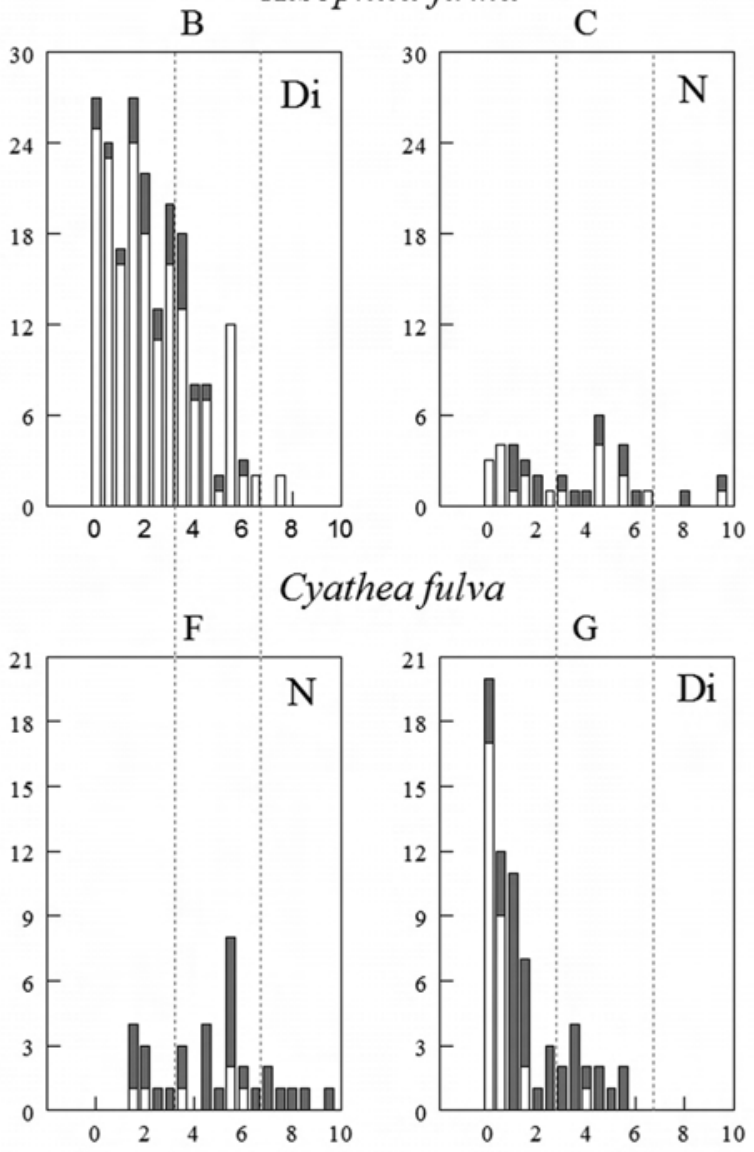

Estériles $\square$ Fértiles
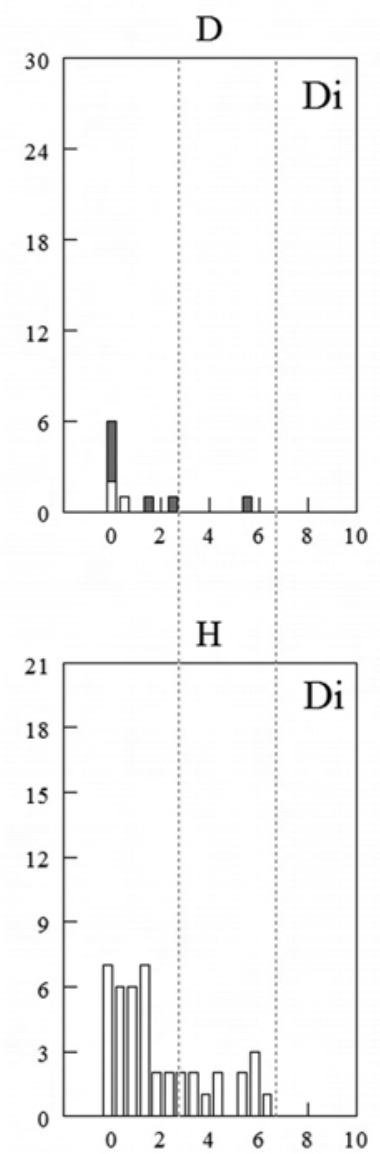

Intervalos de clase de altura (m)

Figura 2. Tipo de poblaciones de Alsophila firma y de Cyathea fulva con base en la altura de los individuos. El Temazate (A) y La Quebradora (B), en Tlanchinol; Los Comales (C) y Los Comales II (D), en San Bartolo Tutotepec; El Temazate (E) y La Bomba (F), en Tlanchinol; Medio Monte $(\mathrm{G})$ y Tutotepec $(\mathrm{H})$, en San Bartolo Tutotepec. Di = dinámica, N = normal.

Relación entre la densidad poblacional y las características del hábitat. El árbol de regresión permitió describir la densidad poblacional en ambas especies de helechos a partir de unas cuantas variables indicadoras. Los resultados derivados del uso de esta técnica en Alsophila firma, indican que la altitud es el factor ambiental más importante en la explicación de los valores observados. La estructura del árbol de regresión señaló dos opciones correlacionadas con una mayor o menor densidad de individuos (Figura 4). La primera opción sugiere que la mayor densidad poblacional se presentó en altitudes menores de 1,668 m, en sitios donde la pendiente fue menor de $52.5^{\circ} \mathrm{y}$ el porcentaje de rocas en el suelo mayor de $67.5 \%$ (Figura 4, flecha con líneas continuas). En los sitios donde la pendiente del terreno fue mayor de $52.5^{\circ}$ la densidad poblacional se redujo drásticamente (27 individuos); en cambio, en los sitios donde el porcentaje de rocas en el suelo fue menor de $67.5 \%$, la densidad disminuyó ligeramente (57.5 individuos). La segunda opción muestra que en los sitios ubicados a más de 1,668 m de altitud y con presencia de caminos o veredas, la densidad poblacional fue baja (Figura 4, flecha con líneas discontinuas).

En Cyathea fulva los resultados fueron diferentes, el árbol de regresión permitió identificar sólo a la cobertura del dosel como la variable más estrechamente relacionada con la densidad poblacional. Es decir, en los sitios donde la cobertura del dosel fue menor de $99.1 \%$, se presentó un número promedio alto de individuos (20.2), pero cuando la cobertura del dosel fue mayor de $99.2 \%$ el número promedio de individuos por sitio resultó bajo: igual o menor de diez. En siete de los 12 sitios analizados, una cobertura del dosel mayor de $90.6 \%$ pero menor de $98.8 \%$, dio como resultado la presencia de 16.1 individuos, en promedio.

Fenología de los individuos marcados. La producción de hojas en los individuos de ambas especies es asincrónica; es decir, se pueden observar hojas en distintas etapas de desarrollo: nuevas, maduras (vegetativas y fértiles) y senescentes, tanto en la época de lluvias (junio-octubre), como 

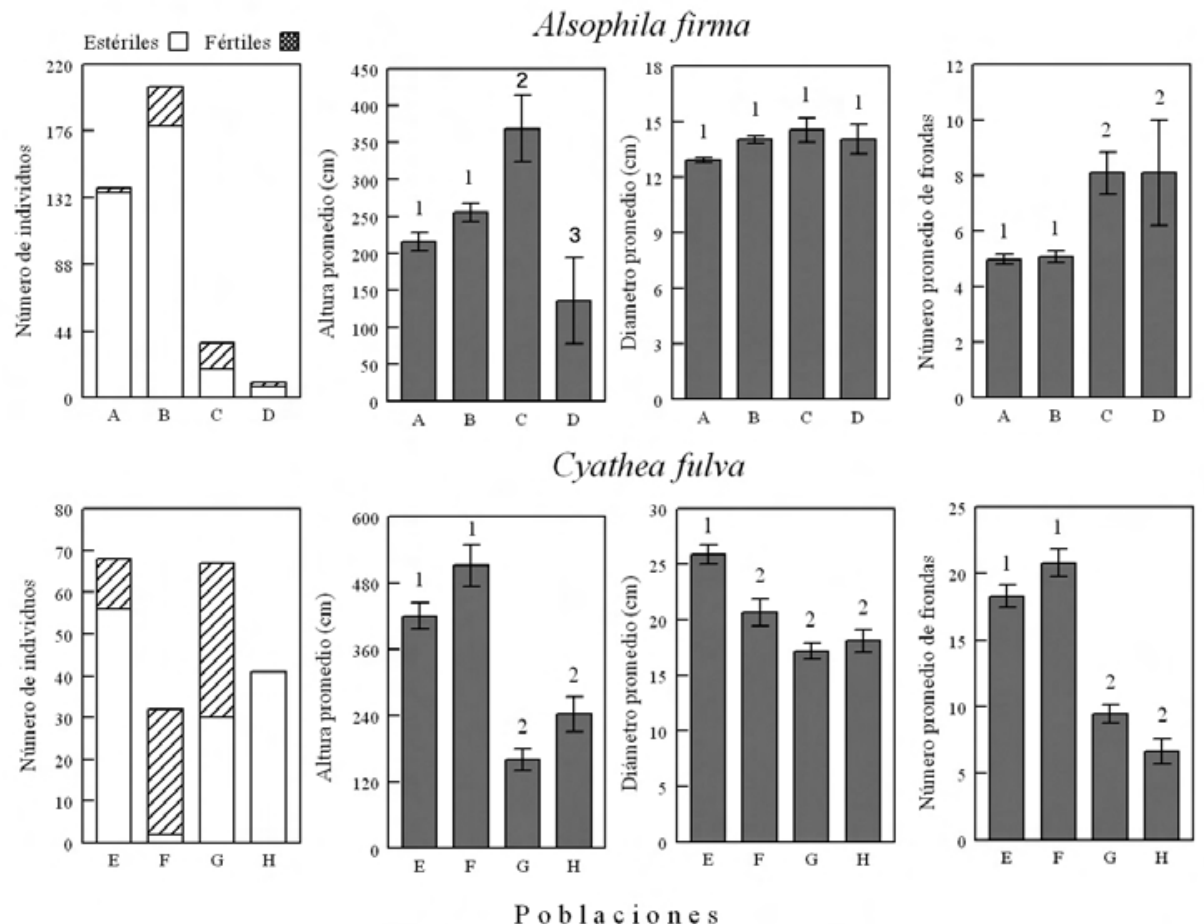

Figura 3. Promedio de los parámetros evaluados dentro de las poblaciones de Alsophila firma y Cyathea fulva en dos municipios del estado de Hidalgo. El Temazate (A) y La Quebradora (B), en Tlanchinol; Los Comales (C) y Los Comales II (D), en San Bartolo Tutotepec; El Temazate (E) y La Bomba (F), en Tlanchinol; Medio Monte (G) y Tutotepec $(\mathrm{H})$, en San Bartolo Tutotepec. Números diferentes sobre cada barra, indican diferencias significativas $(P<0.01)$ entre poblaciones.

en la época de secas (noviembre-mayo). Sin embargo, en Alsophila firma, el número promedio de hojas nuevas (o brotes) fue más elevado en los meses incluidos en la época de secas (marzo y enero), el de hojas maduras en los meses que pertenecen a la época de lluvias (octubre y agosto); y el de hojas senescentes fue independiente de la época del año. Es importante resaltar que las condiciones de humedad y temperatura en el municipio de Tlanchinol permanecieron relativamente estables durante el periodo que comprendió el presente estudio; lo que se evidencia mejor en los valores de precipitación total mensual, mayores de $49 \mathrm{~mm}(4.9 \mathrm{~cm})$ en 13 de los 16 meses considerados (Figura 5).

La fenología foliar fue diferente en Cyathea fulva, dado que el mayor número promedio de hojas nuevas y senescentes ocurrió durante la época de lluvias (junio), y la de hojas maduras en diciembre, mes considerado dentro de la época de secas (Figura 5). De acuerdo con los datos de la estación meteorológica de San Bartolo Tutotepec, los valores de temperatura promedio y precipitación pluvial total mensual difirieron marcadamente entre épocas del año (secas y lluvias), durante el periodo que comprendió el estudio.

El número total de meses que transcurrieron entre dos periodos de máxima producción de hojas nuevas o brotes, difirió entre especies; fue de aproximadamente 11 meses en Alsophila firma y de 13 meses en Cyathea fulva; más del $80 \%$ de los individuos de ambas especies produjeron hojas nuevas durante estos periodos.
Estructura de edades. En los individuos marcados de ambas especies, se estimó que existe una correlación positiva altamente significativa $(P<0.01)$, entre la altura del tallo y el número de cicatrices foliares, una ecuación lineal simple representó adecuadamente esta relación en ambas especies: en Alsophila firma, $\mathrm{r}=0.84(y=9.54 x+2.69)$, y en Cyathea fulva, $\mathrm{r}=0.83(y=4.056 x+6.35)$.

Por otra parte, con base en los datos de altura promedio inicial y final de los tallos, en un intervalo de 12 meses, se estimó que la tasa de crecimiento promedio anual del tallo en Alsophila firma fue de $7.2 \mathrm{~cm}$ y en Cyathea fulva de $10.2 \mathrm{~cm}$. Considerando estos valores, en A. firma, un individuo de 4 $\mathrm{m}$ de altura puede tener una edad de cerca de 46 años, pero de acuerdo con el número de cicatrices foliares su edad sería de alrededor de 31 años. En el caso de $C$. fulva, un individuo de la misma altura $(4 \mathrm{~m})$ tendría, con base en la tasa de crecimiento promedio, 39 años $\mathrm{y}$, por el número de cicatrices foliares, 34 años.

\section{Discusión}

Estructura poblacional. En las dos especies de helechos arborescentes analizadas se encontraron poblaciones normales y dinámicas (tres dinámicas y una normal en $\mathrm{Al}$ sophila firma; dos dinámicas y dos normales en Cyathea fulva). De acuerdo con varios autores, la ocurrencia de poblaciones dinámicas (con predominio de individuos jóve- 


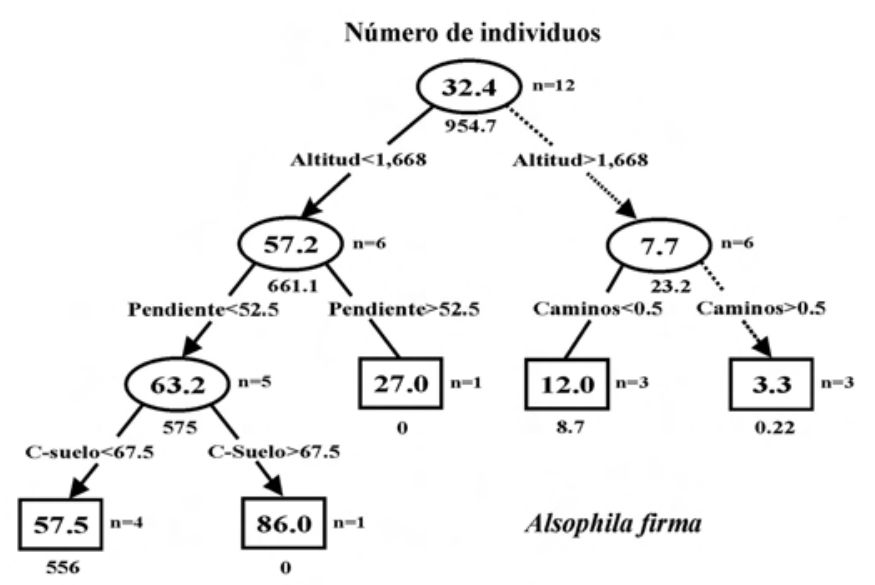

Figura 4. Árbol de regresión para la densidad poblacional en Alsophila firma. Las elipses y los rectángulos representan los nodos no terminales y terminales, respectivamente. Los números dentro de cada elipse o rectángulo indican el promedio del número de individuos de las poblaciones que fluyen por las ramas del árbol hacia un nodo terminal particular. Los números bajo cada elipse o rectángulo indican la suma de cuadrados asociados con el promedio aritmético de todas las muestras que permanecen a través de los nodos. Los valores en las conexiones entre nodos representan los criterios de decisión por medio de los cuales una determinada variable del medio provee las bases para la división. Los vectores con líneas continuas representan la vía que determina la densidad poblacional más alta en el árbol de regresión y los vectores con líneas discontinuas indican la vía que determina la densidad poblacional más baja. C-suelo = Cobertura del suelo con vegetación (\%);

Caminos $=$ presencia de caminos o veredas dentro del bosque.

nes o de tamaño pequeño), en varias especies de helechos arborescentes, por ejemplo en Alsophila auneae (Tanner, 1983), Cyathea spinulosa (Nagano y Suzuki, 2007) y Dicksonia sellowiana (Schmitt et al., 2009), es un indicador de que tienen un alto potencial de regeneración y expansión (Schmitt y Windisch, 2007; Schmitt et al., 2009), y de que se desarrollan bajo condiciones micro-ambientales favorables para el establecimiento, tanto de los gametofitos como de los esporofitos (Bernabe et al., 1999; Mehltreter y García-Franco, 2008).

Sin embargo, este tipo de poblaciones con predominio de individuos jóvenes, también puede ser producto de la acción de otros factores externos: $v$. gr. extracción ilegal de individuos adultos para comercializarlos o para obtener el "maquique", lo que redundaría en la modificación de la estructura de edades de la población, debido a la disminución del número de individuos maduros y viejos (Eleutério y Pérez-Salicrup, 2009).

Mehltreter y García-Franco (2008), encontraron que la baja frecuencia de individuos en una clase de tamaño determinada, en una población de Alsophila firma de Huatusco, Veracruz, puede estar relacionada con eventos o disturbios que ocurrieron en el pasado. La edad de los individuos, estimada conforme al crecimiento promedio anual del tronco, permitió a los autores referidos calcular el tiempo aproximado en el que ocurrió el disturbio. Con base en esta interpretación, una probable explicación de la ausencia de individuos en al menos una clase de tamaño en la mayoría de las poblaciones de A. firma y Cyathea fulva, puede estar relacionada con la ocurrencia de disturbios aislados o eventos estocásticos en diferentes tiempos en cada una de las localidades estudiadas. En la población de A. firma de la localidad Los Comales II en particular, es evidente que las actividades humanas de las últimas décadas (conversión del bosque en potrero), han modificado de manera intermitente la estructura poblacional, pues carece de individuos en varias de las clases de tamaño consideradas.

En el mismo sentido, la ausencia de individuos de menos de $1.5 \mathrm{~m}$ de altura en una de las poblaciones de Cyathea fulva (localidad La Bomba), sugiere que cambios recientes en las condiciones ambientales (tal vez en la última década), han sido desfavorables para el establecimiento de las esporas y/o el desarrollo de las plántulas, dando como resultado una tasa de mortalidad de $100 \%$. Los datos y observaciones en campo apoyan esta hipótesis con respecto a la localidad de la Bomba: la tala ilegal de árboles, la extracción de leña y la apertura de caminos, provocan cambios considerables en la estructura y composición del bosque donde se desarrolla la población de $C$. fulva, que probablemente afecten negativamente la germinación y desarrollo de las plántulas, tal como ha ocurrido con poblaciones de C. australis y Dicksonia antarctica como resultado de la tala controlada de los bosques húmedos del sureste de Australia (Ough y Murphy, 2004).

Con respecto a la existencia de poblaciones normales o estables de helechos arborescentes, caracterizadas por la presencia de individuos en todas las clases de tamaño y con mayor frecuencia en las clases intermedias, puede interpretarse también como un indicador de que las condiciones ambientales locales han permanecido sin cambios apreciables durante varias décadas, lo que da como resultado una tasa constante de reclutamiento de individuos (Large y Braggins, 2004; Schmitt et al., 2009; Sharpe y Mehltreter, 2010).

Todas las poblaciones consideradas como dinámicas de Alsophila firma y Cyathea fulva presentaron valores promedio inferiores de altura de los individuos y número de frondas que las poblaciones normales, por contener un mayor número de organismos jóvenes. Como la producción de hojas nuevas en los helechos arborescentes va acompañada de la elongación del tallo, las plantas de mayor edad son más altas y presentan un mayor número de hojas que las juveniles (Nagano y Susuki, 2007; Mehltreter y GarcíaFranco, 2008).

Se ha documentado que las especies de helechos arborescentes difieren en su tolerancia a diferentes condiciones de luz (Bernabe et al., 1999; Eleutério y Pérez-Salicrup, 2009), se les puede encontrar en los claros del bosque o persis- 

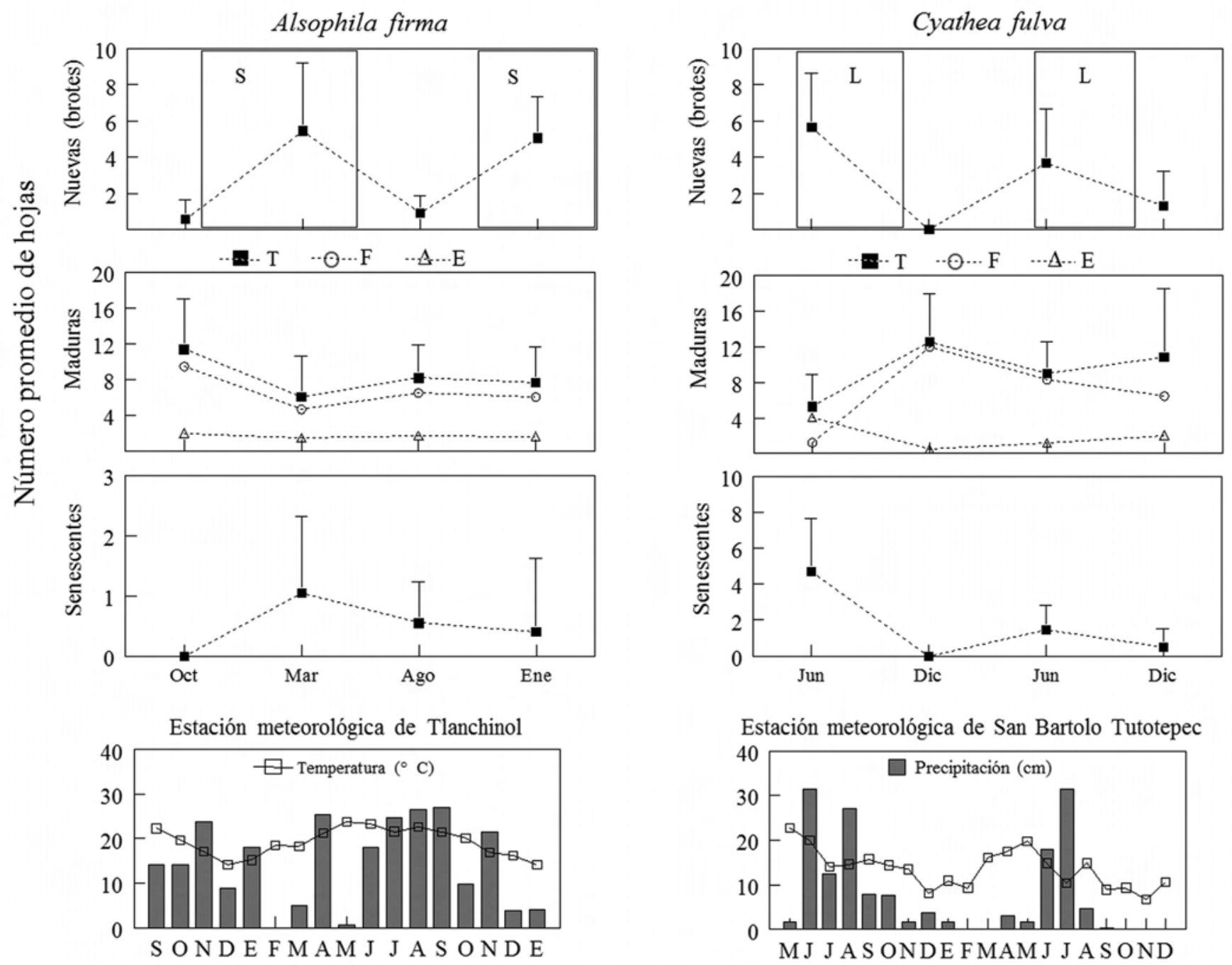

Estación meteorológica de San Bartolo Tutotepec

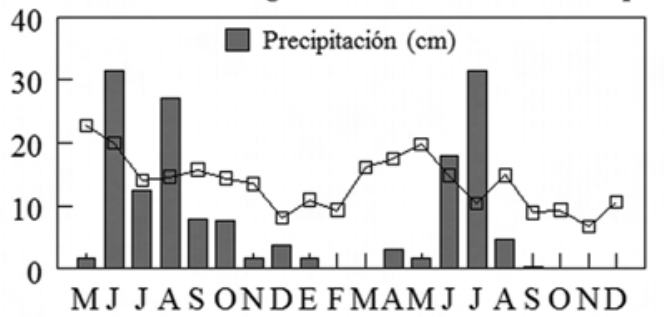

Figura 5. Fenología foliar de Alsophila firma y Cyathea fulva evaluada en 20 individuos de cada especie, seleccionados al azar en los municipios de San Bartolo Tutotepec y de Tlanchinol, estado de Hidalgo. Hojas: T = totales, F = fértiles, E = estériles; temporada o época: $\mathrm{L}=$ lluvias, $\mathrm{S}=$ secas.

tiendo en etapas tardías de la sucesión (Bystriakova et al., 2011). En el presente estudio, todas las poblaciones de ambas especies formaban parte del sub-dosel, en sitios donde la cobertura arbórea fue mayor de $89 \%$, por lo que pueden considerarse como tolerantes a la sombra (esciófilas). Las observaciones en campo y los experimentos de trasplante realizados por Eleutério y Pérez-Salicrup (2009) apoyan la idea de que Alsophila firma y Cyathea fulva se desarrollan mejor en condiciones de sombra que en sitios abiertos. De acuerdo con Arens (2001) y Bystriakova et al. (2011), las especies de helechos tolerantes a la sombra tienen tasas de crecimiento lentas, porque ajustan su metabolismo a las restricciones en la cantidad de luz que reciben desde que germinan y se establecen. Como resultado de ello, sus poblaciones tienden a tener una estructura de tamaños y edades sesgada, con muchos individuos pequeños y pocos altos. La información anterior, concuerda con los resultados obteni- dos en el presente estudio, pues la mayoría de las poblaciones en ambas especies de helechos fueron clasificadas como dinámicas.

El crecimiento del tallo en helechos arborescentes varía ampliamente entre especies y entre hábitats (Schmitt y Windisch, 2012); la relación entre tamaño del tallo y edad es controversial y cuando existe, no es necesariamente lineal, debido principalmente a que la tasa de crecimiento no es constante en todas las etapas del ciclo de vida de los individuos (Schmitt y Windisch, 2006) y a que las condiciones ambientales cambian a través del tiempo (Arens, 2001). Sin embargo, en el presente estudio los individuos de ambas especies presentaron una distribución agrupada en cada población, por lo que es probable que a lo largo de su desarrollo experimentaran condiciones ambientales similares. Como la cobertura del dosel en todas las localidades analizadas fue mayor de $89 \%$, es posible suponer que factores tales como 
la radiación solar, la temperatura del suelo y la humedad, entre otros, han permanecido relativamente homogéneos en los últimos años.

El patrón de distribución gregario en los helechos arborescentes, más evidente a lo largo de arroyos o de sitios con pendientes muy pronunciadas, es común en varios grupos de plantas en los bosques húmedos (Condit et al., 2000; Plotkin et al., 2000; Mehltreter y García-Franco, 2008) y puede atribuirse a la existencia de micro-ambientes idóneos para las plantas, a la tendencia de la mayoría de las esporas o semillas a establecerse cerca de la planta madre (Jones et al., 2007) y a la reproducción vegetativa, que se ha observado en varias especies de Alsophila, donde los troncos suelen ramificar por debajo del suelo (Mehltreter y GarcíaFranco, 2008).

Bystriakova et al. (2011), mencionan que el diámetro del tallo en los helechos arborescentes no aporta una información fidedigna acerca del tamaño de los individuos, porque se mantiene casi constante conforme crecen las plantas. Los resultados del presente estudio concuerdan parcialmente con esta afirmación, debido a que se estimó una correlación lineal significativa entre altura y diámetro, al menos en las primeras etapas de desarrollo (que incluye sólo individuos de tallas pequeñas y medianas), pero es probable que esta relación cambie si se integran datos de individuos de mayor altura en los que el DAP varia muy poco. En las poblaciones analizadas fueron muy escasos los organismos de más de $6 \mathrm{~m}$ de altura.

Relación entre densidad de individuos y ambiente. Las condiciones climáticas en donde se desarrollan las poblaciones de Alsophila firma y Cyathea fulva en el presente estudio, se pueden incluir dentro del intervalo general de variación descrito por Bystriakova et al. (2011) para la mayoría de las especies de Cyatheaceae: tienen un elevado conservadurismo filogenético del nicho con respecto a los climas donde se desarrollan, ya que están restringidas a regiones en donde las temperaturas mínimas raramente están por debajo del punto de congelación y la cantidad de lluvia es alta y esta equitativamente distribuida a lo largo del año.

En el caso de Alsophila firma, en la escala más amplia considerada en el presente estudio (que incluye las poblaciones de los dos municipios), la altitud fue la variable que permitió explicar de la mejor manera, las diferencias en la densidad poblacional. Sin embargo, la altitud es una variable ambiental indirecta, que representa en realidad una combinación compleja de variables, principalmente climáticas (temperatura y precipitación), a las cuales las especies responden (Körner, 2007; Richter, 2008). De acuerdo con esta información, Bystriakova et al. (2011), mencionan que la humedad del aire y del suelo, la temperatura y otras condiciones ambientales que cambian con la altitud, pueden restringir la amplitud del hábitat de las especies de Cyatheaceae con menor capacidad de adaptación.
Los resultados del árbol de regresión indican que las seis poblaciones de Alsophila firma con mayor número de individuos, se encuentran en altitudes de menos de 1,668 m, en la zona de transición del BHM con el bosque tropical (Álvarez-Zúñiga et al., 2012). Como el intervalo de distribución altitudinal más común del BHM de México está entre los 1,500 y 2,500 m (González-Espinosa et al., 2011), es probable que las condiciones climáticas que prevalecen en el límite de distribución altitudinal inferior de este tipo de vegetación, sean favorables para el desarrollo exitoso de las poblaciones de A. firma. Acorde con lo anterior, algunos datos (Luna-Vega, 1997, 2000) y estudios recientes (Mehltreter y García-Franco, 2008; Eleutério y Pérez-Salicrup, 2009), indican que aunque esta especie es común en el BHM, también puede desarrollarse en climas más cálidos, característicos del bosque tropical sub-caducifolio.

Las condiciones climáticas son consideradas como los principales factores limitantes de la distribución y abundancia de los helechos a escala continental y regional. Sin embargo, a una escala local, otros factores ambientales además de los climáticos, como por ejemplo edáficos y topográficos, también pueden influir en las características de las poblaciones de helechos (Landi y Angiolini, 2008; AlfonsoMoreno et al., 2011). Los resultados del presente estudio son consistentes con esta afirmación, dado que a escala local (cuando se incluye sólo el subconjunto de poblaciones con mayor número de individuos), la pendiente del terreno y la cobertura del suelo fueron las variables que mejor explicaron las diferencias en los valores de densidad poblacional en Alsophila firma.

Con respecto a Cyathea fulva, la cobertura del dosel fue la única variable relacionada con la densidad poblacional; este resultado llama la atención porque el intervalo de variación de la cobertura arbórea fluctuó entre 89 y $99.5 \%$. Sin embargo, el árbol de regresión detectó que dentro de este pequeño intervalo de entrada de luz pueden ocurrir cambios significativos en la densidad poblacional de esta especie. De cualquier forma estos resultados deben tomarse con reserva, en el sentido de que los árboles de regresión representan herramientas estadísticas sumamente útiles para hacer inferencias ecológicas y para generar hipótesis que deben comprobarse en estudios experimentales posteriores (Sánchez-González y López-Mata, 2005; Kallimanis et al., 2007; Zhang y Liu, 2012).

Las adaptaciones a diferentes cantidades de luz han sido analizadas en varias especies de helechos arborescentes. En el caso particular del género Cyathea se han encontrado resultados contrastantes: en algunas de las especies el tallo crece más rápido y produce más frondas y esporas en condiciones de luminosidad (Arens y Sánchez, 2000; Arens, 2001), e incluso se ha observado que en $C$. caracasana no hay reclutamiento de esporofitos cuando el dosel es cerrado (Arens, 2001). En cambio, otras especies de Cyatheaceae ( $v$. gr. Alsophila bryophila, A. firma, C. fulva y C. pubescens) 
Estructura poblacional y háBitat de dos especies de Cyatheaceae

son consideradas como tolerantes, ya que son capaces de germinar, crecer y producir esporas bajo el dosel del bosque (Large y Braggins, 2004; Eleutério y Pérez-Salicrup, 2009; Bystriakova et al., 2011). Los resultados del presente estudio indican que, aunque $C$. fulva es una especie tolerante a la sombra, requiere de un poco de luz para establecerse y desarrollarse; pues cuando el dosel es cerrado, la densidad de individuos es baja. Bernabe et al. (1999) describen un comportamiento semejante para $C$. pubescens.

Recientemente, se ha señalado que la tolerancia a la sombra está determinada por numerosos factores bióticos $(v$. gr. la ontogenia de las plantas) y abióticos, entre los que sobresalen la disponibilidad de agua y nutrientes (Valladares y Niinemets, 2008). Acorde con lo anterior, en las dos especies de helechos arborescentes analizadas, se presentan adaptaciones fisiológicas y estructurales relacionadas con las condiciones de escasez de luz y elevada humedad (higrofilia) donde se desarrollan: hojas grandes, relación tallo y raíz desproporcionada a favor del primero y alta resistencia al flujo de agua (Watkins et al., 2010).

Fenología foliar y edad de los individuos marcados. En las dos especies analizadas, la presencia de hojas nuevas, maduras (vegetativas y fértiles) y senescentes ocurrió durante todo el año, debido a la heterogeneidad en las respuestas a nivel individual. Este patrón continuo en la fenología foliar ha sido observado en varias especies de helechos arborescentes: Cyathea pubescens (Tanner, 1983) en Jamaica, $C$. spinulosa (Nagano y Suzuki, 2007) en Japón, Alsophila firma (Mehltreter y García-Franco, 2008) en México, y C. delgadii (Schmitt y Windisch, 2007) y Dicksonia sellowiana (Schmitt et al., 2009) en Brasil.

De cualquier forma, el número promedio de hojas nuevas que se produjeron en las dos especies difirió con respecto a la época del año. En Alsophila firma se registró en marzo de 2011 y en enero de 2012 (meses con poca precipitación pluvial). En cambio, en Cyathea fulva la mayor producción de hojas nuevas fue en junio de 2011 y en junio de 2012 (meses con alta precipitación pluvial; INIFAP, 2013). En otras especies de helechos arborescentes, como C. pubescens (Tanner, 1983) y C. spinulosa (Nagano y Suzuki, 2007), el mayor número de hojas nuevas se produce en primavera y verano; en A. firma ocurre sincrónicamente entre junio y septiembre (Mehltreter y García-Franco, 2008) y en C. atrovirens entre junio y noviembre, pero con un máximo en el mes de septiembre (Schmitt y Windisch, 2012).

De acuerdo con los datos anteriores, en casi todas las especies referidas, la mayor producción de hojas nuevas ocurrió en los meses con precipitación pluvial alta (entre junio y noviembre), excepto en la población de Alsophila firma de la localidad del Temazate. Esta respuesta "asincrónica" en la producción de hojas nuevas puede deberse a que no hubo una temporada de sequía bien definida durante el tiempo en que se desarrolló el estudio. Las lluvias ocurrieron en prác- ticamente todos los meses del año en el municipio de Tlanchinol (INIFAP, 2013), como la humedad no fue un factor limitante para el desarrollo de los individuos de A. firma, es probable que otras variables abióticas (como la temperatura y la longitud del día) y/o bióticas ( $v . g r$. competencia interespecifica con Cyathea fulva), estén influyendo sobre la fenología foliar, como se ha sugerido en otras investigaciones (Schmitt y Windisch, 2012).

En las dos técnicas utilizadas para estimar la edad de los helechos arborescentes se requiere de un monitoreo a largo plazo para que los resultados sean confiables, porque el incremento en la longitud del tallo y la producción de hojas puede diferir significativamente entre años consecutivos debido a cambios en las condiciones ambientales (Mehltreter y García-Franco, 2008; Ramírez-Valencia et al., 2009; Sharpe y Mehltreter, 2010; Schmitt y Windisch, 2012).

Aunque el tiempo que abarcó el estudio de la fenología foliar en ambas especies de helechos arborescentes (menos de 18 meses), es insuficiente para obtener una estimación confiable de la edad de los individuos, un resultado interesante es que se observó una relación lineal estadísticamente significativa entre la altura de los individuos y el número de cicatrices foliares. El conteo de estas estructuras permitió determinar una edad razonable para los individuos de Alsophila firma y Cyathea fulva, considerando que los tiempos de vida estimados se encuentran dentro del intervalo de valores propuesto para otras especies de Cyatheaceae en distintas regiones del planeta (Sharpe y Mehltreter, 2010; Bystriakova et al., 2011; Schmitt y Windisch, 2012).

El crecimiento anual del tallo fue lento (de menos de $10 \mathrm{~cm}$ ) en ambas especies, lo que concuerda con los valores estimados para otras especies de Alsophila, Cyathea y Dicksonia tolerantes a la sombra (Arens y Sánchez, 2000; Ramírez-Valencia et al., 2009; Bystriakova et al., 2011), lo cual se debe principalmente a la escasez de luz solar disponible para los individuos. Acorde con lo antes mencionado, algunos autores opinan que las especies tolerantes a la sombra, A. bryophila (Large y Braggins, 2004), A. firma y $C$. fulva (Eleutério y Pérez Salicrup, 2009), pueden mantener una tasa de crecimiento lenta pero constante bajo el dosel del bosque.

El conocimiento de cómo el tamaño y la estructura de las poblaciones están relacionados con las condiciones ambientales es particularmente relevante en el caso de Alsophila firma y Cyathea fulva, debido a que ambas especies están protegidas por la legislación mexicana (SEMARNAT, 2010), incluidas en la categoría de "sujetas a protección especial". Los resultados obtenidos en el presente estudio indican que el establecimiento y supervivencia a largo plazo de las poblaciones, de ambas especies de helechos arborescentes, depende en gran medida de la conservación del BHM, debido a que, por su capacidad de tolerancia a la sombra, requieren de la existencia de cierto grado de cobertura vegetal. 


\section{Agradecimientos}

Al Consejo Nacional de Ciencia y Tecnología por la beca de posgrado (254578) otorgada a la primera autora. Esta investigación contó con apoyo económico parcial para el trabajo en campo del proyecto "Estructura, diversidad de especies vegetales y distribución actual de los bosques de haya (Fagus grandifolia subsp. mexicana) en el estado de Hidalgo, México", propuesta 169141, Investigación Científica Básica, Consejo Nacional de Ciencia y Tecnología; y del proyecto FOMIX-Hidalgo titulado "Diversidad Biológica del estado de Hidalgo" 191908 (tercera etapa). Los autores agradecen ampliamente los comentarios y sugerencias del Dr. Klaus Mehltreter y de un revisor anónimo, los cuales contribuyeron a mejorar sustancialmente el contenido del presente artículo.

\section{Literatura citada}

Aguraiuja R., Zobel M., Zobel K. y Moora M. 2008. Conservation of the endemic fern lineage Diellia (Aspleniaceae) on the Hawaiian Islands: can population structure indicate regional dynamics and endangering factors? Folia Geobotanica 43:3-18.

Alfonso-Moreno R.A., Cadena-Vargas C.E., Morales G., Peña N. y Pérez B. 2011. Conservación integral de Dicksonia sellowiana Hook., en Bogotá D.C. y su área de influencia. Revista de la Academia Colombiana de Ciencias 35:79-96.

Álvarez-Zúñiga E., Sánchez-González A., López-Mata L. y TejeroDíez J.D. 2012. Composición y abundancia de las pteridofitas en el bosque mesófilo de montaña del municipio de Tlanchinol, Hidalgo, México. Botanical Sciences 90:163-177.

Arens N.C. 2001. Variation in performance of the tree fern Cyathea caracasana (Cyatheaceae) across a successional mosaic in an Andean cloud forest. American Journal of Botany 88:545-551.

Arens N.C. y Sánchez B.P. 2000. Variation in tree fern stipe length with canopy height: tracking preferred habitat through morphological change. American Fern Journal 90:1-15.

Bernabe N., Williams-Linera G. y Palacios-Rios M. 1999. Tree ferns in the interior and at the edge of a Mexican cloud forest remnant: spore germination and sporophyte survival and establishment. Biotropica 31:83-88.

Bystriakova N., Schneider H. y Coomes D. 2011. Evolution of the climatic niche in scaly tree ferns (Cyatheaceae, Polypodiopsida). Botanical Journal of the Linnean Society 165:1-19.

CITES. Convención sobre el Comercio Internacional de Especies Amenazadas de Fauna y Flora Silvestres. 2010. < http://www.cites.org/eng/disc/species.php > (consultado 21 de junio de 2012).

Condit R., Ashton P.S., Baker P., Bunyavejchewin S., Gunatilleke S., Gunatilleke N., Hubbell S.P., Foster R.B., Itoh A., LaFrankie J.V., Lee H.S., Losos E., Manokaran N., Sukumar R. y Yamakura T. 2000. Spatial patterns in the distribution of tropical tree species. Science 288:1414-1418.

Cruz-Cárdenas G., Villaseñor J.L., López-Mata L. y Ortíz E. 2012. Potential distribution of humid mountain forest in Mexico. Botanical Sciences 90:331-340.

Eleutério A.A. y Pérez-Salicrup D. 2009. Transplanting tree ferns to promote their conservation in Mexico. American Fern Journal 99:279-291.
Hanski I. y Simberloff D. 1997. The metapopulation approach, its history, conceptual domain, and application to conservation. En: Hanski I. y Gilpin M.E. Eds. Metapopulation Biology, pp. 5-26, Academic Press, San Diego.

INIFAP. Instituto Nacional de Investigaciones Forestales, Agrícolas y Pecuarias. 2013. Red Nacional de Estaciones Estatales Agroclimatológicas.<http://clima.inifap.gob.mx/redclima/historicos.aspx > (consultada 31 de enero de 2013).

IUCN. International Union for Conservation of Nature and Natural Resources. 2011. IUCN Red List of Threatened Species. <www.iucnredlist.org> (consultada el 12 de marzo de 2011).

Jones M.M., Olivas R.P., Tuomisto H. y Clark D.B. 2007. Environmental and neighbourhood effects on tree fern distributions in a Neotropical lowland rain forest. Journal of Vegetation Science 18:13-24.

Kallimanis A.S., Ragia V., Sgardelis S.P. y Pantis J.D. 2007. Using regression trees to predict alpha diversity based upon geographical and habitat characteristics. Biodiversity and Conservation 16:3863-3876.

Kingston N., Waldren S. y Smyth N. 2004. Conservation genetics and ecology of Angiopteris chauliodonta Copel. (Marattiaceae), a critically endangered fern from Pitcairn Island, South Central Pacific Ocean. Biological Conservation 117:309-319.

Körner C. 2007. The use of 'altitude' in ecological research. Trends in Ecology and Evolution 22:569-574.

Landi M. y Angiolini C. 2008. Habitat characteristics and vegetation context of Osmunda regalis L. at the southern edge of its distribution in Europe. Botanica Helvetica 118:45-57.

Large M.F. y Braggins J.E. 2004. Tree ferns. Timber Press, Portland.

Luna-Vega M.I. 1997. Florística y biogeografía de algunos bosques mesófilos de la Huasteca Hidalguense: Fase I (Tenango de Doria y Tlanchinol). Universidad Nacional Autónoma de México. Facultad de Ciencias. Bases de datos SNIB-CONABIO. Proyecto No. B133. México, D.F.

Luna-Vega M.I. 2000. Florística y biogeografía de algunos bosques mesófilos de la Huasteca Hidalguense: Fase 3 (Chapulhuacán y Pisaflores). Universidad Nacional Autónoma de México. Facultad de Ciencias. Bases de datos SNIB2010-CONABIO. Proyecto No. L091. México, D.F.

Mehltreter K. 2010. Fern Conservation. En: Mehltreter K., Walker L.R. y Sharpe M.J. Eds. Fern Ecology, pp. 323-359, Cambridge University Press, Nueva York.

Mehltreter K. y García-Franco J.G. 2008. Leaf phenology and trunk growth of the deciduous tree fern Alsophila firma (Baker) D. S. Conant in a lower montane mexican forest. American Fern Journal 98:1-13.

Nagano T. y Suzuki E. 2007. Leaf demography and growth pattern of the tree fern Cyathea spinulosa in Yakushima Island. Tropics 16:47-57.

Ough K. y Murphy A. 2004. Decline in tree-fern abundance after clearfell harvesting. Forest Ecology and Management 199:153-163.

Pavón N.P. y Meza S.M. 2009. Cambio climático en el estado de Hidalgo: clasificación y tendencias climáticas. Universidad Autónoma del Estado de Hidalgo. Pachuca.

Pérez-Paredes M.G., Sánchez-González A. y Tejero-Díez J. D. 2012. Listado de licopodios y helechos del municipio de Zacualtipán de Ángeles, Hidalgo, México. Polibotánica 33:57-73.

Plotkin J.B., Potts M.D., Leslie N., Manokaran N., LaFrankie J. y 
Ashton P.S. 2000. Species-area curves, spatial aggregation, and habitat specialization in tropical forests. Journal of Theoretical Biology 207:81-99.

Ponce-Reyes R., Reynoso-Rosales V.H., Watson .J.E.M, VanDerWal J., Fuller R.A., Pressey R.L. y Possingham H.P. 2012. Vulnerability of cloud forest reserves in Mexico to climate change. Nature Climate Change 2:448-452.

Ramírez-Barahona S., Luna-Vega I. y Tejero-Díez D. 2011. Species richness, endemism, and conservation of American tree ferns (Cyatheales). Biodiversity and Conservation 20:59-72.

Ramírez-Valencia V., Sanín D y Álvarez-Mejía L.M. 2009. Estimación del crecimiento de Dicksonia sellowiana Hook., (Dicksoniaceae Hook.), en la Reserva Forestal Protectora de Río Blanco, Manizales, Caldas, y registros colombianos de su fertilidad. Boletín Científico Museo de Historia Natural Universidad de Caldas 13:17-29.

Richter M. 2008. Tropical Mountain forests - distribution and general features. Biodiversity and Ecology Series 2:7-24.

Rodríguez-Ramírez E.Ch., Sánchez-González A. y Ángeles-Pérez G. 2013. Current distribution and coverage of Mexican beech forests Fagus grandifolia subsp. mexicana in Mexico. Endangered Species Research 20:205-216.

Rzedowski J. 2006. Vegetación de México. 1ra. Edición digital. Comisión Nacional para el Conocimiento y Uso de la Biodiversidad, México, México, D.F.

Sánchez-González A. y López-Mata L. 2005. Plant species richness and diversity along an altitudinal gradient in the Sierra Nevada, Mexico. Diversity and Distributions 11:567-575.

Schmitt J.L. y Windisch P.G. 2006. Growth rates and age estimates of Alsophila setosa Kaulf. in Southern Brazil. American Fern Journal 96:103-111.

Schmitt J.L. y Windisch P.G. 2007. Estrutura populacional e desenvolvimento da fase esporofitica de Cyathea delgadii Sternb. (Cyatheaceae, Monilophyta) no sul do Brasil. Acta Botanica Brasilica 21:731-740.

Schmitt J.L. y Windisch P.G. 2012. Caudex growth and phenology of Cyathea atrovirens (Langsd. \& Fisch.) Domin (Cyatheaceae) in secondary forest, southern Brazil. Brazilian Journal of Biology 72:397-405.

Schmitt J.L., Schneider P.H. y Windisch P.G. 2009. Crescimento do cáudice e fenologia de Dicksonia sellowiana Hook. (Dicksoniaceae) no sul do Brasil. Acta Botanica Brasilica 23:282-291.

SEMARNAT. Secretaría de Medio Ambiente y Recursos Naturales. 2010. Norma Oficial Mexicana NOM-059-SEMARNAT-2010. Protección ambiental-Especies nativas de México de flora y fauna silvestres-Categorías de riesgo y especificaciones para su inclusión, exclusión o cambio-Lista de especies en riesgo.

Sharpe J.M. y Mehltreter K. 2010. Ecological insights from fern population dynamics. En: Mehltreter K., Walker L.R. y Sharpe J.M. Eds. Fern Ecology, pp. 61-110, Cambridge University Press, New York.

StatSoft, Inc. 2010. STATISTICA (data analysis software system), version 7. www.statsoft.com.

Tanner E.V.J. 1983. Leaf demography and growth of tree-fern Cyathea pubescens Mett. ex Kuhn in Jamaica. Botanical Journal of the Linnean Society 87:213-227.

Valladares F. y Niinemets Ü. 2008. Shade tolerance, a key plant feature of complex nature and consequences. Annual Review of Ecology, Evolution, and Systematics 39:237-257.

Vandermeer J.H. y Goldberg D.E. 2003. Population Ecology First Principles. Princeton University Press, Nueva Jersey.

Villaseñor J.L. 2010. El bosque húmedo de montaña en México y sus plantas vasculares: catálogo florístico-taxonómico. Universidad Nacional Autónoma de México. Comisión Nacional para el Conocimiento y Uso de la Biodiversidad. México, D.F.

Watkins J.E.Jr., Holbrook N.M. y Zwieniecki M.A. 2010. Hydraulic properties of fern sporophytes: consequences for ecological and evolutionary diversification. American Journal of Botany 97:2007-2019.

Zhang R. y Liu T. 2012. Plant species diversity and community classification in the southern Gurbantunggut Desert. Acta Ecologica Sinica 32:6056-6066.

Recibido: 3 de abril de 2013

Aceptado: 5 de julio de 2013 